\title{
COVID-19
}

\section{Health Evidence Summary No.114}

\section{Kerry Millington}

Liverpool School of Tropical Medicine (LSTM)

01 March 2021

This weekly COVID-19 health evidence summary (HES) is based on 3.5 hours of desk-based research. The summary is not intended to be a comprehensive summary of available evidence on COVID-19 but aims to make original documents easily accessible to decision makers which, if relevant to them, they should go to before making decisions.

\section{Clinical characteristics and management}

\begin{tabular}{|c|c|c|c|c|}
\hline $\begin{array}{l}\text { Publication } \\
\text { date }\end{array}$ & Title/URL & $\begin{array}{l}\text { Journal/Article } \\
\text { type }\end{array}$ & Summary & Keywords \\
\hline 23.02.2021 & $\begin{array}{l}\text { Signs and } \\
\text { symptoms } \\
\text { to determine } \\
\text { if a patient } \\
\text { presenting } \\
\text { in primary } \\
\text { care or } \\
\text { hospital } \\
\text { outpatient } \\
\text { settings has } \\
\text { COVID-19 }\end{array}$ & $\begin{array}{l}\text { Cochrane | } \\
\text { Systematic } \\
\text { Review } \\
\text { (update to the } \\
\text { July } 2020 \\
\text { review) }\end{array}$ & $\begin{array}{l}\text { Based on currently } \\
\text { available data, neither } \\
\text { absence nor presence of } \\
\text { signs or symptoms are } \\
\text { accurate enough to rule in } \\
\text { or rule out COVID-19 } \\
44 \text { relevant studies with } \\
26,884 \text { participants were } \\
\text { included. There were no } \\
\text { studies specifically on } \\
\text { children, and only one } \\
\text { focussed on older adults } \\
\text { Most studies were } \\
\text { conducted in hospital } \\
\text { settings so results may not } \\
\text { be representative of } \\
\text { primary care settings. } \\
\text { Results do not apply to } \\
\text { children or older adults } \\
\text { and do not differentiate } \\
\text { between disease } \\
\text { severities } \\
\text { A single symptom or sign } \\
\text { cannot accurately } \\
\text { diagnose COVID-19 but } \\
\text { the presence of anosmia, } \\
\text { ageusia, fever or cough, } \\
\text { could be useful to identify } \\
\text { people for further testing }\end{array}$ & $\begin{array}{l}\text { Symptoms, } \\
\text { signs, } \\
\text { COVID-19 }\end{array}$ \\
\hline
\end{tabular}




\begin{tabular}{|c|c|c|c|c|}
\hline & & & $\begin{array}{l}\text { Prospective studies in an } \\
\text { unselected population } \\
\text { presenting to primary care } \\
\text { or hospital outpatients } \\
\text { settings, examining } \\
\text { combinations of signs and } \\
\text { symptoms to evaluate the } \\
\text { syndromic presentation of } \\
\text { COVID-19 remain urgent - } \\
\text { findings of which could } \\
\text { inform subsequent } \\
\text { management decisions }\end{array}$ & \\
\hline 23.02.2021 & $\begin{array}{l}\text { Identification } \\
\text { and } \\
\text { validation of } \\
\text { clinical } \\
\text { phenotypes } \\
\text { with } \\
\text { prognostic } \\
\text { implications } \\
\text { in patients } \\
\text { admitted to } \\
\text { hospital with } \\
\text { CovID-19: } \\
\text { a } \\
\text { multicentre } \\
\text { cohort study }\end{array}$ & $\begin{array}{l}\text { The Lancet } \\
\text { Infectious } \\
\text { Diseases | } \\
\text { Article }\end{array}$ & $\begin{array}{l}\text { - Investigation of the } \\
\text { existence and } \\
\text { characterisation of clinical } \\
\text { phenotypes for COVID-19 } \\
\text { patients at hospital } \\
\text { admission } \\
\text { - distinct clinical } \\
\text { phenotypes, based on } \\
\text { demographics, underlying } \\
\text { conditions, clinical and } \\
\text { laboratory data and } \\
\text { radiological features at } \\
\text { presentation of patients } \\
\text { admitted to hospital with } \\
\text { COVID-19, were } \\
\text { associated with patient } \\
\text { prognosis and correlate } \\
\text { with mortality } \\
\text { Authors have developed } \\
\text { and validated a simplified } \\
\text { probabilistic model for } \\
\text { phenotype assignment } \\
\text { available as a tool online } \\
\text { Identifying COVID-19 } \\
\text { phenotypes could allow } \\
\text { better pathogenesis- } \\
\text { targeted approaches for } \\
\text { therapies in the design } \\
\text { and selection of } \\
\text { participants in clinical trials } \\
\text { and be helpful in clinical } \\
\text { management by } \\
\text { identifying low-risk } \\
\text { patients and patients who } \\
\text { may need closer } \\
\text { monitoring during } \\
\text { admission } \\
\end{array}$ & $\begin{array}{l}\text { Clinical } \\
\text { phenotypes, } \\
\text { prognosis }\end{array}$ \\
\hline
\end{tabular}




\section{Epidemiology and modelling}

\begin{tabular}{|c|c|c|c|c|}
\hline $\begin{array}{l}\text { Publication } \\
\text { date }\end{array}$ & Title/URL & $\begin{array}{l}\text { Journal/Article } \\
\text { type }\end{array}$ & Summary & Keywords \\
\hline 24.02.2021 & $\begin{array}{l}\text { Increased } \\
\text { transmission } \\
\text { of SARS- } \\
\text { CoV-2 } \\
\text { lineage } \\
\text { B.1.1.7 } \\
\text { (VOC } \\
2020212 / 01 \text { ) } \\
\text { is not } \\
\text { accounted } \\
\text { for by a } \\
\text { replicative } \\
\text { advantage } \\
\text { in primary } \\
\text { airway cells } \\
\text { or antibody } \\
\text { escape }\end{array}$ & $\begin{array}{l}\text { bioRxiv | pre- } \\
\text { print (not peer } \\
\text { reviewed) }\end{array}$ & $\begin{array}{l}\text { - Increased transmission of } \\
\text { B.1.1.7 (VoC 202012/01) } \\
\text { is not caused by } \\
\text { increased replication (as } \\
\text { measured on human } \\
\text { airway epithelial cells) or } \\
\text { escape from serological } \\
\text { immunity }\end{array}$ & $\begin{array}{l}\text { UK variant, } \\
\text { transmission }\end{array}$ \\
\hline
\end{tabular}

\section{Infection Prevention and Control}

\begin{tabular}{|c|c|c|c|c|}
\hline $\begin{array}{l}\text { Publication } \\
\text { date }\end{array}$ & Title/URL & $\begin{array}{l}\text { Journal/Article } \\
\text { type }\end{array}$ & Summary & Keywords \\
\hline 24.02.2021 & $\begin{array}{l}\text { COVID-19: } \\
\text { lessons and } \\
\text { experiences } \\
\text { from South } \\
\text { Africa's first } \\
\text { surge }\end{array}$ & $\begin{array}{l}\text { BMJ Global } \\
\text { Health | } \\
\text { Practice }\end{array}$ & $\begin{array}{l}\text { - Swift countrywide lockdowns } \\
\text { enabled increased health } \\
\text { system capacity but also } \\
\text { resulted in economic } \\
\text { hardship especially for the } \\
\text { most vulnerable } \\
\text { During a health emergency, } \\
\text { country leaders must } \\
\text { maintain a unified front with } \\
\text { continuous and accurate } \\
\text { communication with the } \\
\text { public to maintain trust with } \\
\text { ongoing accountability } \\
\text { among leaders and to avoid } \\
\text { corruption } \\
\text { Epidemiological data is } \\
\text { critical to inform types of } \\
\text { intervention and where }\end{array}$ & $\begin{array}{l}\text { Lockdown, } \\
\text { lessons, } \\
\text { South } \\
\text { Africa }\end{array}$ \\
\hline
\end{tabular}


- Healthcare workers and communities should be empowered with technology

\section{Therapeutics}

\begin{tabular}{|c|c|c|c|c|}
\hline $\begin{array}{l}\text { Publication } \\
\text { date }\end{array}$ & Title/URL & $\begin{array}{l}\text { Journal/Articl } \\
\text { e type }\end{array}$ & Summary & Keywords \\
\hline 25.02.2021 & $\begin{array}{l}\text { Dexamethaso } \\
\text { ne in } \\
\text { hospitalized } \\
\text { patients with } \\
\text { Covid-19 }\end{array}$ & $\begin{array}{l}\text { NEJM | } \\
\text { Original } \\
\text { Article }\end{array}$ & $\begin{array}{l}\text { Final results of } \\
\text { a controlled, } \\
\text { open-label trial } \\
\text { comparing a } \\
\text { range of } \\
\text { possible } \\
\text { treatments in } \\
\text { patients who } \\
\text { were } \\
\text { hospitalised } \\
\text { with Covid-19 } \\
\text { randomly } \\
\text { assigned to } \\
\text { receive oral or } \\
\text { intravenous } \\
\text { dexamethason } \\
\text { e for up to } 10 \\
\text { days (2104 } \\
\text { patients) or to } \\
\text { receive usual } \\
\text { standard care } \\
\text { (4321 patients) } \\
\text { Use of } \\
\text { dexamethason } \\
\text { e resulted in } \\
\text { lower } 28-d a y \\
\text { mortality in } \\
\text { patients } \\
\text { hospitalised } \\
\text { with Covid-19 } \\
\text { who were } \\
\text { receiving } \\
\text { either invasive } \\
\text { mechanical } \\
\text { ventilation or } \\
\text { oxygen alone } \\
\text { at } \\
\text { randomisation } \\
\text { but not among } \\
\text { those receiving } \\
\text { no respiratory } \\
\text { support }\end{array}$ & $\begin{array}{l}\text { Dexamethasone, } \\
\text { severe COVID-19, } \\
\text { mortality }\end{array}$ \\
\hline
\end{tabular}




\begin{tabular}{|c|c|c|c|c|}
\hline 25.02.2021 & $\begin{array}{l}\text { Interleukin-6 } \\
\text { Receptor } \\
\text { Antagonists } \\
\text { in Critically III } \\
\text { Patients with } \\
\text { Covid-19 }\end{array}$ & $\begin{array}{l}\text { NEJM | } \\
\text { Original } \\
\text { Article }\end{array}$ & $\begin{array}{l}\text { Evaluation of } \\
\text { tocilizumab } \\
\text { and sarilumab } \\
\text { in an ongoing } \\
\text { international, } \\
\text { multifactorial, } \\
\text { adaptive } \\
\text { platform trial } \\
\text { (REMAP-CAP) } \\
\text { Adult patients } \\
\text { with Covid-19 } \\
\text { were randomly } \\
\text { assigned to } \\
\text { receive } \\
\text { tocilizumab, } \\
\text { sarilumab or } \\
\text { standard care } \\
\text { within } 24 \mathrm{~h} \\
\text { after starting } \\
\text { organ support } \\
\text { in the intensive } \\
\text { care unit } \\
\text { In critically ill } \\
\text { patients with } \\
\text { Covid-19 } \\
\text { receiving } \\
\text { organ support } \\
\text { in ICUs, } \\
\text { treatment with } \\
\text { the IL-6 } \\
\text { receptor } \\
\text { antagonists } \\
\text { tocilizumab } \\
\text { and sarilumab } \\
\text { improved } \\
\text { outcomes, } \\
\text { including } \\
\text { survival } \\
\end{array}$ & $\begin{array}{l}\text { Tocilizumab, sarilum } \\
\text { ab, severe COVID-19 }\end{array}$ \\
\hline 25.02.2021 & $\begin{array}{l}\text { Tocilizumab } \\
\text { in } \\
\text { hospitalized } \\
\text { patients with } \\
\text { severe Covid- } \\
19 \\
\text { pneumonia }\end{array}$ & $\begin{array}{l}\text { NEJM | } \\
\text { Original } \\
\text { Article }\end{array}$ & $\begin{array}{l}\text { Covid-19 is } \\
\text { associated } \\
\text { with immune } \\
\text { dysfunction } \\
\text { including } \\
\text { elevated } \\
\text { interleukin-6 } \\
\text { levels. Use of } \\
\text { tocilizumab, a } \\
\text { monoclonal } \\
\text { antibody } \\
\text { against the IL- } \\
6 \text { receptor, has } \\
\text { been shown in } \\
\text { case reports } \\
\text { and } \\
\text { retrospective }\end{array}$ & $\begin{array}{l}\text { Tocilizumab, severe } \\
\text { COVID-19 }\end{array}$ \\
\hline
\end{tabular}




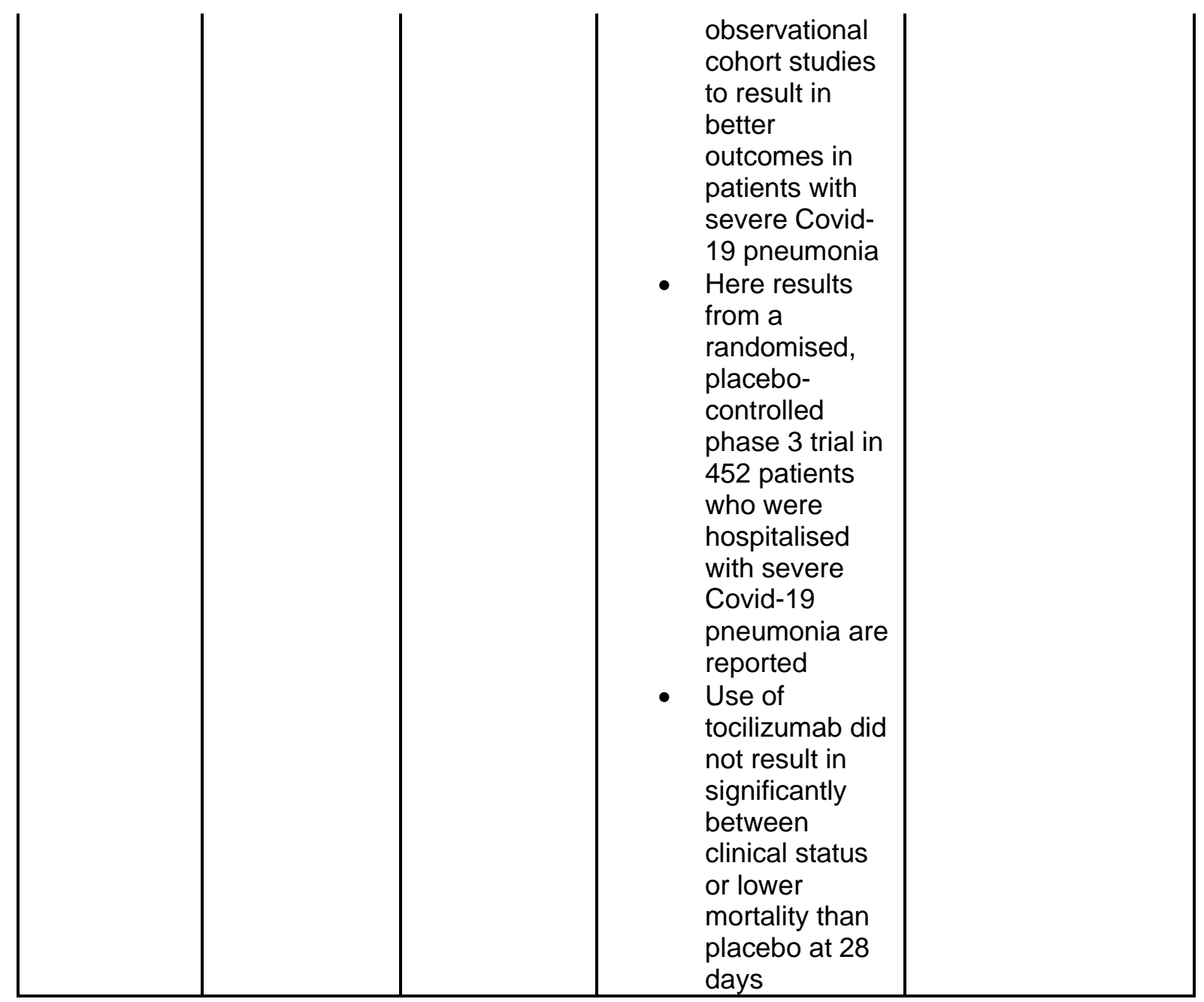

\section{Vaccines}

\begin{tabular}{|c|c|c|c|c|}
\hline $\begin{array}{l}\text { Publication } \\
\text { date }\end{array}$ & Title/URL & $\begin{array}{l}\text { Journal/Article } \\
\text { type }\end{array}$ & Summary & Keywords \\
\hline 24.02.2021 & $\begin{array}{l}\text { BNT162b2 } \\
\text { mRNA } \\
\text { Covid-19 } \\
\text { vaccine in a } \\
\text { nationwide } \\
\text { mass } \\
\text { vaccination } \\
\text { setting }\end{array}$ & $\begin{array}{l}\text { NEJM | } \\
\text { Original } \\
\text { Article }\end{array}$ & 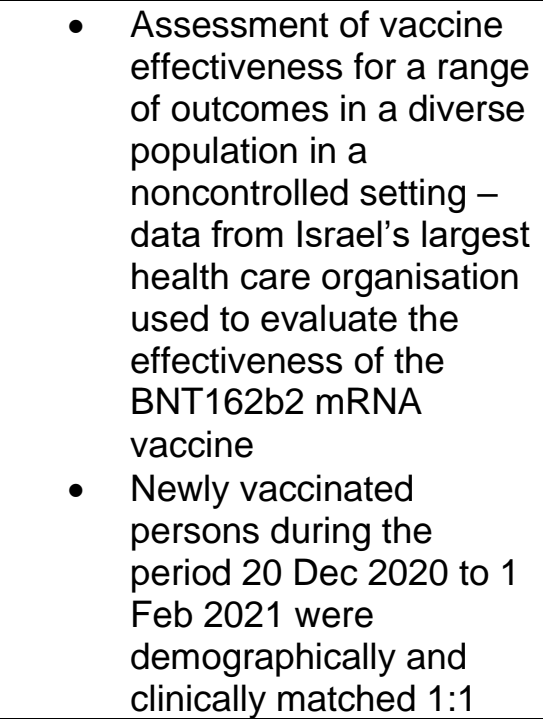 & $\begin{array}{l}\text { Pfizer- } \\
\text { BioNTech } \\
\text { vaccine, } \\
\text { effectiveness, } \\
\text { Israel, real- } \\
\text { world }\end{array}$ \\
\hline
\end{tabular}




$\mid$\begin{tabular}{|l|l|}
$\mid$ & with unvaccinated \\
controls \\
Vaccine effectiveness \\
was estimated for each \\
of the following study \\
outcomes at days 14 to \\
20 after the first dose \\
and at 7 or more days \\
after the second dose: \\
SARS-CoV-2 infection, \\
symptomatic Covid-19, \\
Covid-19-related \\
hospitalisation, severe \\
illness and death. Each \\
study group included \\
596.618 persons \\
This study in a \\
nationwide mass \\
vaccination setting \\
suggests that the \\
BNT162b2 mRNA \\
vaccine is effective for a \\
wide range of Covid-19 \\
related outcomes - \\
consistent with \\
randomised trial findings
\end{tabular}

\section{Indirect impact of COVID-19}

\begin{tabular}{|c|c|c|c|c|}
\hline $\begin{array}{l}\text { Publication } \\
\text { date }\end{array}$ & Title/URL & $\begin{array}{l}\text { Journal/Article } \\
\text { type }\end{array}$ & Summary & Keywords \\
\hline 25.02.2021 & $\begin{array}{l}\text { A double- } \\
\text { edged } \\
\text { sword- } \\
\text { telemedicine } \\
\text { for maternal } \\
\text { care during } \\
\text { COVID-19: } \\
\text { findings } \\
\text { from a } \\
\text { global } \\
\text { mixed- } \\
\text { methods } \\
\text { study of } \\
\text { healthcare } \\
\text { providers }\end{array}$ & $\begin{array}{l}\text { BMJ Global } \\
\text { Health | } \\
\text { Original } \\
\text { Research }\end{array}$ & $\begin{array}{l}\text { Telemedicine has been } \\
\text { rapidly applied globally } \\
\text { to address disruptions of } \\
\text { healthcare provision } \\
\text { during the COVID-19 } \\
\text { pandemic } \\
\text { Here the second round } \\
\text { of a global online survey } \\
\text { documents the } \\
\text { experiences of providing } \\
\text { telemedicine for } \\
\text { maternal and newborn } \\
\text { healthcare during the } \\
\text { pandemic among } \\
\text { healthcare professionals } \\
\text { globally } \\
\text { Maternal and newborn } \\
\text { healthcare providers } \\
\text { globally considered } \\
\text { telemedicine of benefit } \\
\text { during the pandemic for }\end{array}$ & $\begin{array}{l}\text { Telemedicine, } \\
\text { maternal and } \\
\text { newborn care }\end{array}$ \\
\hline
\end{tabular}




\begin{tabular}{|c|c|c|c|c|c|}
\hline & & & & $\begin{array}{l}\text { different components of } \\
\text { maternal and newborn } \\
\text { healthcare but rapid } \\
\text { adaptation was not } \\
\text { optimally supported by } \\
\text { guidelines, training, } \\
\text { adequate equipment, } \\
\text { reimbursement for cost } \\
\text { of connectivity and } \\
\text { insurance payments for } \\
\text { care provided remotely } \\
\text { Also, predominantly in } \\
\text { LMICs, substantial } \\
\text { groups of families were } \\
\text { not able to be reached } \\
\text { by telemedicine } \\
\text { encountering different } \\
\text { barriers in providing } \\
\text { high-quality maternity } \\
\text { care by } \\
\text { telemedicine including } \\
\text { internet connection } \\
\text { problems, lack of } \\
\text { necessary equipment, } \\
\text { digital illiteracy and } \\
\text { distrust } \\
\text { Authors recommend that } \\
\text { more research regarding } \\
\text { the effectiveness, } \\
\text { efficacy and quality of } \\
\text { telemedicine for } \\
\text { maternal healthcare in } \\
\text { difference contexts is } \\
\text { needed before long-term } \\
\text { adaptations in care } \\
\text { provision away from } \\
\text { face-to-face interactions } \\
\text { Guidelines for care } \\
\text { provision and } \\
\text { approaches are need to } \\
\text { minimise socioeconomic } \\
\text { and technological } \\
\text { inequalities in access to } \\
\text { care }\end{array}$ & \\
\hline 23.02.2021 & $\begin{array}{l}\text { COVID-19 in } \\
\text { the Global } \\
\text { South: } \\
\text { Impacts and } \\
\text { policy } \\
\text { responses }\end{array}$ & $\begin{array}{l}\text { Southern } \\
\text { Voice | } \\
\text { Occasional } \\
\text { Paper Series } \\
\text { No. } 69\end{array}$ & & $\begin{array}{l}\text { Analysis exploring the } \\
\text { social and economic } \\
\text { impact of, and } \\
\text { responses to, COVID-19 } \\
\text { and how it is affecting } \\
\text { the Global South }\end{array}$ & $\begin{array}{l}\text { social and } \\
\text { economic } \\
\text { impact, } \\
\text { Global South }\end{array}$ \\
\hline
\end{tabular}


Social Science

\begin{tabular}{|c|c|c|c|c|}
\hline $\begin{array}{l}\text { Publication } \\
\text { date }\end{array}$ & Title/URL & $\begin{array}{l}\text { Journal/Article } \\
\text { type }\end{array}$ & Summary & Keywords \\
\hline 24.02.2021 & $\begin{array}{l}\text { An } \\
\text { intersection } \\
\text { al human } \\
\text { rights } \\
\text { approach } \\
\text { to } \\
\text { prioritising } \\
\text { access to } \\
\text { COVID-19 } \\
\text { vaccines }\end{array}$ & $\begin{array}{l}\text { BMJ Global } \\
\text { Health | } \\
\text { Analysis }\end{array}$ & $\begin{array}{l}\text { - International human } \\
\text { rights law requires that } \\
\text { if 'vulnerability' is used } \\
\text { as a criterion for } \\
\text { priority access to } \\
\text { COVID-19 vaccines, } \\
\text { this must include social } \\
\text { vulnerability (e.g. } \\
\text { socioeconomic status) } \\
\text { as well as medical } \\
\text { vulnerability (e.g. } \\
\text { comorbidities) and } \\
\text { broader consideration } \\
\text { of intersectional needs } \\
\text { in society and the } \\
\text { disproportionate impact } \\
\text { that COVID-19 is } \\
\text { having on population } \\
\text { groups with pre- } \\
\text { existing social and } \\
\text { medical vulnerabilities } \\
\text { Here authors state that } \\
\text { existing } \\
\text { frameworks/mechanis } \\
\text { ms and proposals for } \\
\text { COVID-19 vaccine } \\
\text { allocation have } \\
\text { shortcomings from a } \\
\text { human rights } \\
\text { perspective with } \\
\text { national vaccine } \\
\text { allocation schemes } \\
\text { needing to adopt a } \\
\text { more intersectional } \\
\text { approach allocating } \\
\text { COVID-19 vaccines on } \\
\text { health, social and } \\
\text { financial } \\
\text { vulnerabilities }\end{array}$ & $\begin{array}{l}\text { Vaccine } \\
\text { prioritisation, } \\
\text { human rights, } \\
\text { intersectional } \\
\text {, medical and } \\
\text { social } \\
\text { vulnerability }\end{array}$ \\
\hline
\end{tabular}




\section{Comments, Editorials, Opinions, Blogs, News}

\begin{tabular}{|c|c|c|}
\hline $\begin{array}{l}\text { Publication } \\
\text { date }\end{array}$ & Title/URL & Journal | Article type \\
\hline 01.03 .2021 & $\begin{array}{l}\text { Supporting COVID-19 Recovery and Improving } \\
\text { Health Outcomes: The Case for Health Taxes }\end{array}$ & CGD | Blog \\
\hline March 2021 & Feelings towards COVID-19 vaccination in Africa & $\begin{array}{l}\text { The Lancet Infection } \\
\text { Diseases | Newsdesk }\end{array}$ \\
\hline March 2021 & $\begin{array}{l}\text { COVID-19 hindering progress against female } \\
\text { genital mutilation }\end{array}$ & $\begin{array}{l}\text { The Lancet Public Health | } \\
\text { Editorial }\end{array}$ \\
\hline March 2021 & $\begin{array}{l}\text { Migrant health is public health: a call for equitable } \\
\text { access to COVID-19 vaccines }\end{array}$ & $\begin{array}{l}\text { The Lancet Public Health | } \\
\text { Correspondence }\end{array}$ \\
\hline 01.03 .2021 & $\begin{array}{l}\text { COVID-19 research in critical care: the good, the } \\
\text { bad, and the ugly }\end{array}$ & Intensive Care Medicine \\
\hline 01.03 .2021 & India's COVID-19 vaccine roll-out in a nutshell & Southern Voice | Blog \\
\hline 27.02 .2021 & New WTO leader faces COVID-19 challenges & The Lancet | World Report \\
\hline 27.02 .2021 & Just allocation of COVID-19 vaccines & $\begin{array}{l}\text { BMJ Global Health I } \\
\text { Editorial }\end{array}$ \\
\hline 27.02.2021 & $\begin{array}{l}\text { FDA Issues Emergency Use Authroization for third } \\
\text { COVID-19 Vaccine }\end{array}$ & FDA | News \\
\hline 26.02 .2021 & An expert explains variants & Wellcome | News \\
\hline 25.02 .2021 & $\begin{array}{l}\text { Interleukin-6 Reception Inhibition in Covid-19 - } \\
\text { cooling the inflammatory soup }\end{array}$ & NEJM | Editorial \\
\hline 25.02 .2021 & Research in the context of a pandemic & NEJM | Editorial \\
\hline 25.02 .2021 & The RECOVERY Platform & NEJM | Editorial \\
\hline
\end{tabular}




\begin{tabular}{|l|l|l|} 
24.02.2021 & $\begin{array}{l}\text { Major rise in public support for COVID vaccine - } \\
\text { Oxford study }\end{array}$ & Oxford University | News \\
\hline 24.02.2021 & $\begin{array}{l}\text { COVID-19 drug practices risk antimicrobial } \\
\text { resistance evolution }\end{array}$ & The Lancet Microbe \\
\hline 23.02.2021 & $\begin{array}{l}\text { Who gets a COVID-19 vaccine and who pays? } \\
\text { The need for economic analysis }\end{array}$ & CGD | Blog \\
\hline 19.02.2021 & $\begin{array}{l}\text { Creating effective research-policy partnerships for } \\
\text { COVID response and beyond }\end{array}$ & CGD | Blog \\
\hline 02.02.2021 & $\begin{array}{l}\text { Comparing the Covid-19 vaccines developed by } \\
\text { Pfizer, Moderna, and Johnson \& Johnson }\end{array}$ & STAT | News \\
\hline
\end{tabular}




\section{Dashboards \& Trackers}

\begin{tabular}{|c|c|c|c|c|c|c|c|}
\hline $\begin{array}{l}\text { Cases \& deaths: } \\
\text { Global }\end{array}$ & $\begin{array}{l}\text { Cases \& } \\
\text { deaths: } \\
\text { Regional }\end{array}$ & $\begin{array}{l}\text { Cases \& } \quad \& \\
\text { deaths: } \\
\text { Country }\end{array}$ & $\begin{array}{l}\text { Living evidence \& } \\
\text { policy maps }\end{array}$ & $\begin{array}{l}\text { Current research } \\
\text { including trials }\end{array}$ & Diagnostics & Treatments & Vaccines \\
\hline WHO sitreps & WHO Africa & Ghana & COVID-NMA & WHO & $\begin{array}{l}\text { FIND SARS- } \\
\text { CoV-2 Test } \\
\text { Tracker }\end{array}$ & $\begin{array}{l}\text { Global COVID- } \\
19 \text { Clinical Trial } \\
\text { Tracker }\end{array}$ & CEPI \\
\hline WHO dashboard & $\begin{array}{l}\text { African } \\
\text { Arguments }\end{array}$ & Indonesia & EPPI Centre & $\begin{array}{l}\text { WHO International } \\
\text { Clinical Trials } \\
\text { Registry Platform } \\
\text { (ICTRP) }\end{array}$ & $\begin{array}{l}\text { FIND SARS- } \\
\text { CoV-2 } \\
\text { Diagnostics: } \\
\text { performance } \\
\text { data }\end{array}$ & $\begin{array}{l}\text { US NIH } \\
\text { registered } \\
\text { clinical trials }\end{array}$ & $\begin{array}{l}\text { Vaccine Centre } \\
\text { LSHTM }\end{array}$ \\
\hline $\begin{array}{l}\text { Johns Hopkins } \\
\text { University }\end{array}$ & $\begin{array}{l}\text { European } \\
\text { CDC }\end{array}$ & Nigeria CDC & $\begin{array}{l}\text { Norwegian } \\
\text { Institute of Public } \\
\text { Health }\end{array}$ & Cytel & $\begin{array}{l}\text { Serology-based } \\
\text { tests for COVID- } \\
19\end{array}$ & Solidarity trial & $\begin{array}{l}\text { COVID-19 } \\
\text { Oxford Vaccine } \\
\text { Trial }\end{array}$ \\
\hline WEF & & Sierra Leone & $\begin{array}{l}\text { Oxford C19 } \\
\text { Government } \\
\text { Response } \\
\text { Tracker } \\
\text { (OxCGRT) }\end{array}$ & US NIH & $\begin{array}{l}\text { Our World in } \\
\text { Data: C19 } \\
\text { Testing }\end{array}$ & $\begin{array}{l}\text { COVID-19 } \\
\text { Therapeutics } \\
\text { Accelerator }\end{array}$ & $\begin{array}{l}\text { COVID-19 } \\
\text { Vaccine Tracker }\end{array}$ \\
\hline
\end{tabular}




\begin{tabular}{|c|c|c|c|c|}
\hline $\begin{array}{l}\text { Our World in } \\
\text { Data }\end{array}$ & Singapore & $\begin{array}{l}\text { Our World in } \\
\text { Data: C19 Policy } \\
\text { responses }\end{array}$ & COVID-evidence & $\begin{array}{l}\text { Our World in } \\
\text { Data: COVID- } \\
19 \text { vaccinations }\end{array}$ \\
\hline Global 5050 & UK & $\begin{array}{l}\text { IFPRI COVID-19 } \\
\text { Policy Response } \\
\text { Portal }\end{array}$ & Cochrane & \\
\hline $\begin{array}{l}\text { CEBM, } \\
\text { University of } \\
\text { Oxford }\end{array}$ & US & $\begin{array}{l}\text { COVID-19 } \\
\text { Primer }\end{array}$ & Clinicaltrials.gov & \\
\hline $\begin{array}{l}\text { Humanitarian } \\
\text { Data Exchange }\end{array}$ & & NIH LitCovid & UKCDR & \\
\hline $\begin{array}{l}\text { Information is } \\
\text { Beautiful }\end{array}$ & & $\begin{array}{l}\text { WHO COVID-19 } \\
\text { Database }\end{array}$ & & \\
\hline LSHTM & & & & \\
\hline $\begin{array}{l}\text { HealthMap } \\
\text { (cases) }\end{array}$ & & & & \\
\hline $\begin{array}{l}\text { The Commons } \\
\text { Project }\end{array}$ & & & & \\
\hline SeroTracker & & & & \\
\hline
\end{tabular}




\section{C19 Resource Hubs}

\begin{tabular}{|c|c|c|c|c|c|}
\hline Global & $\begin{array}{l}\text { Regional } \\
\text { \& Country }\end{array}$ & $\begin{array}{l}\text { Academic } \\
\text { journals \& } \\
\text { Publishers }\end{array}$ & $\begin{array}{l}\text { Institutes/Centres/ } \\
\text { Funders/Other }\end{array}$ & Health Topics & Social Sciences \\
\hline $\begin{array}{l}\text { WHO } \\
\text { COVID-19 } \\
\text { pandemic }\end{array}$ & $\begin{array}{l}\text { Africa } \\
\text { CDC }\end{array}$ & $\begin{array}{l}\text { Annals of } \\
\text { Internal } \\
\text { Medicine }\end{array}$ & LSTM & $\begin{array}{l}\text { Stop TB } \\
\text { Partnership }\end{array}$ & SSHAP \\
\hline $\begin{array}{l}\text { WHO risk } \\
\text { communicati } \\
\text { on }\end{array}$ & $\begin{array}{l}\text { African } \\
\text { Union }\end{array}$ & BMJ & LSHTM & & IDA \\
\hline WHO Q\&A & $\begin{array}{l}\text { Nigeria } \\
\text { CDC }\end{array}$ & $\begin{array}{l}\text { Bulletin of } \\
\text { the WHO }\end{array}$ & $\begin{array}{l}\text { ICL MRC Centre } \\
\text { for Global } \\
\text { Infectious Disease } \\
\text { Analysis }\end{array}$ & $\begin{array}{l}\text { Global } \\
\text { Menstrual } \\
\text { Collective }\end{array}$ & $\begin{array}{l}\text { Disability and } \\
\text { inclusion }\end{array}$ \\
\hline $\begin{array}{l}\text { WHO Global } \\
\text { research }\end{array}$ & $\begin{array}{l}\text { GeoPoll: } \\
\text { SSA }\end{array}$ & $\begin{array}{l}\text { Cambridge } \\
\text { University } \\
\text { Press }\end{array}$ & ODI & $\begin{array}{l}\text { SLH: } \\
\text { Handwashing } \\
\text { in low } \\
\text { resource } \\
\text { settings }\end{array}$ & $\begin{array}{l}\text { Coregroup } \\
\text { IDDC }\end{array}$ \\
\hline $\begin{array}{l}\text { COVID-19 } \\
\text { Solidarity } \\
\text { Response } \\
\text { Fund }\end{array}$ & $\begin{array}{l}\text { Global } \\
\text { Health } \\
\text { Network } \\
\text { Africa }\end{array}$ & Cell Press & $\begin{array}{l}\text { Johns Hopkins } \\
\text { University }\end{array}$ & $\begin{array}{l}\text { RBM } \\
\text { Partnership }\end{array}$ & $\begin{array}{l}\text { Ethics, health } \\
\text { systems \& } \\
\text { COVID-19 }\end{array}$ \\
\hline UN & $\begin{array}{l}\text { African } \\
\text { Academy } \\
\text { of } \\
\text { Sciences }\end{array}$ & Cochrane & $\begin{array}{l}\text { Center for Global } \\
\text { Development }\end{array}$ & $\begin{array}{l}\text { Epidemic } \\
\text { Preparedness } \\
\text { Innovations }\end{array}$ & $\begin{array}{l}\text { Social } \\
\text { Development } \\
\text { Direct C19 blog } \\
\text { series }\end{array}$ \\
\hline UN Women & $\begin{array}{l}\text { Africa } \\
\text { Evidence } \\
\text { Network }\end{array}$ & Elsevier & $\begin{array}{l}\text { CMMID } \\
\text { Repository }\end{array}$ & $\begin{array}{l}\text { Southern } \\
\text { Voice }\end{array}$ & \\
\hline
\end{tabular}




\begin{tabular}{|c|c|c|c|c|c|}
\hline UNOCHA & $\begin{array}{l}\text { OCHA } \\
\text { Southern } \\
\text { and } \\
\text { Eastern } \\
\text { Africa } \\
\text { COVID-19 } \\
\text { Digest }\end{array}$ & $\begin{array}{l}\text { Health } \\
\text { Policy and } \\
\text { Planning }\end{array}$ & $\begin{array}{l}\text { Norwegian } \\
\text { Institute of Public } \\
\text { Health }\end{array}$ & & \\
\hline UNHCR & $\begin{array}{l}\text { South } \\
\text { African } \\
\text { Governme } \\
\text { nt }\end{array}$ & $\begin{array}{l}\text { JAMA } \\
\text { Network }\end{array}$ & $\begin{array}{l}\text { Oxford Centre for } \\
\text { Evidence-based } \\
\text { Medicine }\end{array}$ & & \\
\hline UNICEF & & The Lancet & HEART & & \\
\hline UNESCO & & $\begin{array}{l}\text { medRxiv } \\
\text { and } \\
\text { bioRxiv } \\
\text { (Preprints) }\end{array}$ & UKRI & & \\
\hline UN WFP & & NEJM & Evidence Aid & & \\
\hline GOARN & & $\begin{array}{l}\text { Oxford } \\
\text { University } \\
\text { Press }\end{array}$ & $\mathrm{NIH}$ & & \\
\hline EPI-WIN & & PLoS & $\begin{array}{l}\text { IFPRI Resources } \\
\text { and Analyses of } \\
\text { C19 Impact }\end{array}$ & & \\
\hline World Bank & & $\begin{array}{l}\text { SAGE } \\
\text { journals }\end{array}$ & $\begin{array}{l}\text { Prevent } \\
\text { Epidemics }\end{array}$ & & \\
\hline $\begin{array}{l}\text { Our World in } \\
\text { Data }\end{array}$ & & Science & & & \\
\hline $\begin{array}{l}\text { COVID-19 } \\
\text { Narratives by } \\
\text { David } \\
\text { Nabarro }\end{array}$ & & $\begin{array}{l}\text { Springer } \\
\text { Nature }\end{array}$ & & & \\
\hline
\end{tabular}




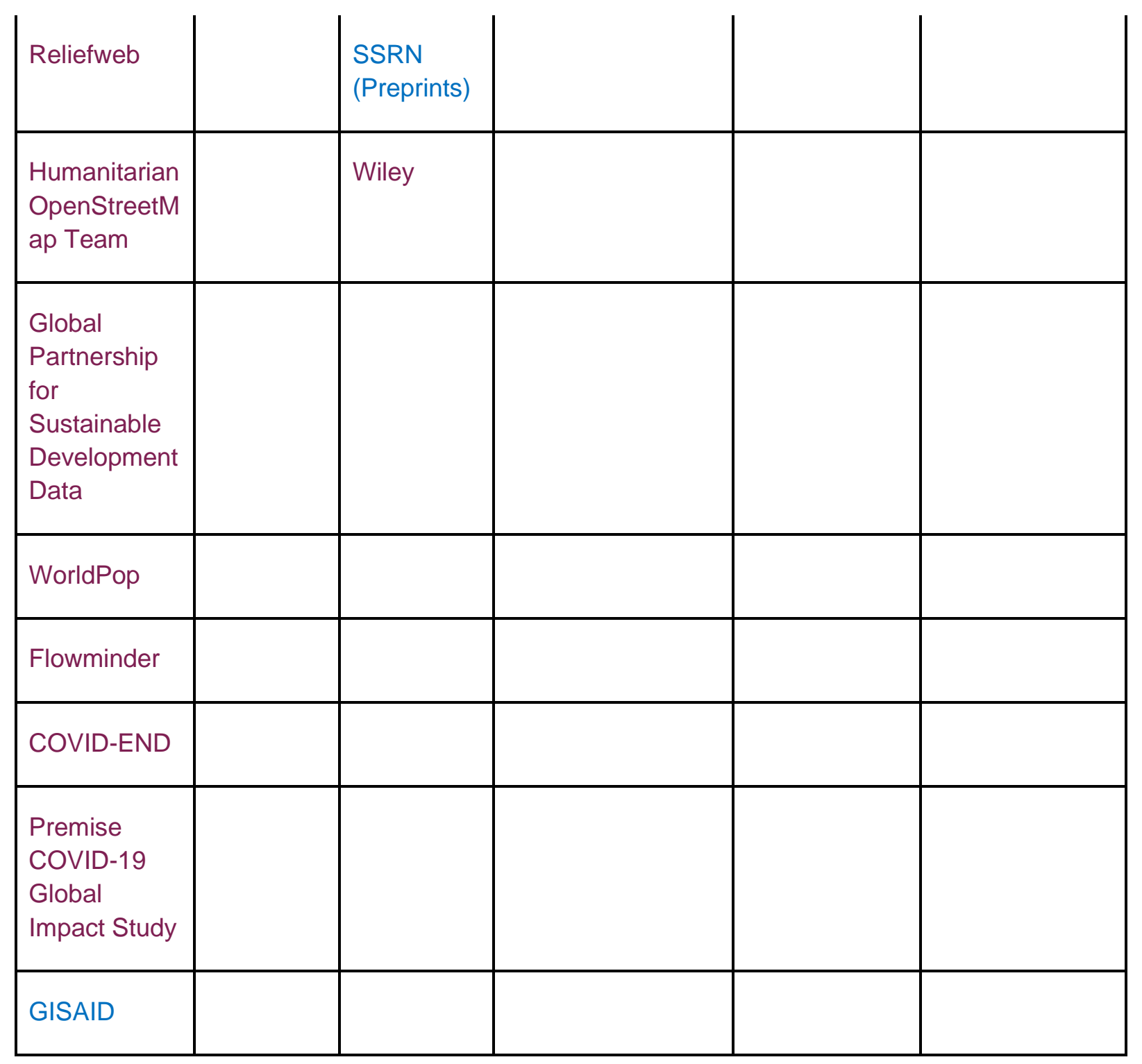

\section{Online learning \& events}

\begin{tabular}{|l|l|l|l|l|}
\hline Date & Title/URL & $\begin{array}{l}\text { Online } \\
\text { learning/event }\end{array}$ & Duration & Lead \\
\hline $\begin{array}{l}8 \mathrm{March} \\
2021\end{array}$ & $\begin{array}{l}\text { Chronic Respiratory } \\
\text { Diseases in the } \\
\text { COVID era }\end{array}$ & Webinar & & GARD, WHO \\
\hline $\begin{array}{l}\text { February } \\
2021\end{array}$ & $\begin{array}{l}\text { COVID-19 } \\
\text { vaccination training } \\
\text { for health workers }\end{array}$ & Online training & $3 \mathrm{~h}$ & WHO \\
\hline
\end{tabular}




\begin{tabular}{|c|c|c|c|c|}
\hline 14.01 .2021 & $\begin{array}{l}\text { Evidence to impact in } \\
\text { crisis: how have we } \\
\text { measured up during } \\
\text { the COVID-19 } \\
\text { pandemic? }\end{array}$ & Webinar & $1 \mathrm{~h} 30$ & CGD \\
\hline 04.12 .2020 & $\begin{array}{l}\text { COVID-19, supply } \\
\text { chain resilience and } \\
\text { global trade }\end{array}$ & Webinar & $1 \mathrm{~h}$ & CGD \\
\hline 03.12 .2020 & $\begin{array}{l}\text { More money for } \\
\text { health services: What } \\
\text { is the tole of PFM in } \\
\text { the "new normal"? }\end{array}$ & $\begin{array}{l}\text { WHO \& CGD } \\
\text { Health systems } \\
\text { Governance \& } \\
\text { Financing }\end{array}$ & $1 \mathrm{~h} 30$ & Joe Kutzin \\
\hline 01.12 .2020 & $\begin{array}{l}\text { Solutions and support } \\
\text { for the mental } \\
\text { wellbeing of } \\
\text { community health } \\
\text { workers on the } \\
\text { COVID-19 frontline }\end{array}$ & Webinar & & $\begin{array}{l}\text { HSG TWG on CHWs } \\
\text { with The George } \\
\text { Institute for Global } \\
\text { Health }\end{array}$ \\
\hline 19.11 .2020 & $\begin{array}{l}\text { Looking at the } \\
\text { pandemic with a } \\
\text { gender lens }\end{array}$ & $\begin{array}{l}\text { Live Twitter } \\
\text { conversation }\end{array}$ & & SSHAP \\
\hline 16.11 .2020 & $\begin{array}{l}\text { HIFA and WHO } \\
\text { collaborate to } \\
\text { promote sharing of } \\
\text { experience and } \\
\text { expertise around the } \\
\text { maintenance of } \\
\text { essential health } \\
\text { services during (and } \\
\text { after) the pandemic }\end{array}$ & $\begin{array}{l}\text { 4-week } \\
\text { discussion } \\
\text { starting } 16 \text { Nov }\end{array}$ & & HIFA \\
\hline 10.11 .2020 & $\begin{array}{l}\text { COVID-19 vaccine } \\
\text { predictions part } 2 \text { : } \\
\text { estimating the time } \\
\text { before we approve } \\
\text { efficacious COVID-19 } \\
\text { vaccines }\end{array}$ & Online event & $1 \mathrm{~h} 30$ & CGD \\
\hline
\end{tabular}




\begin{tabular}{|c|c|c|c|c|}
\hline 16.10 .2020 & $\begin{array}{l}\text { Financing a Global } \\
\text { Public Health } \\
\text { Response }\end{array}$ & Online event & $1 \mathrm{~h} 30$ & CGD \\
\hline 02.10 .2020 & $\begin{array}{l}\text { Understanding and } \\
\text { Improving COVID-19 } \\
\text { Vaccine Portfolio }\end{array}$ & Online event & $1 \mathrm{~h} 30$ & CGD \\
\hline 21.09 .2020 & $\begin{array}{l}\text { Mitigating the } \\
\text { Economic and Health } \\
\text { Impact of COVID-19 } \\
\text { across Africa }\end{array}$ & Online event & $1 \mathrm{~h} 30$ & CGD, GF, AU \\
\hline June 2020 & $\begin{array}{l}\text { OpenWHO, the free, } \\
\text { open-access learning } \\
\text { platform for health } \\
\text { emergencies, now } \\
\text { offers } 10 \text { online } \\
\text { courses related to } \\
\text { COVID19. }\end{array}$ & Online courses & Varies & WHO \\
\hline $\begin{array}{l}\text { Available } \\
\text { now }\end{array}$ & $\begin{array}{l}\text { Standard } \\
\text { precautions: } \\
\text { Environmental } \\
\text { cleaning and } \\
\text { disinfection }\end{array}$ & Online course & 1 hour & WHO \\
\hline $\begin{array}{l}\text { Available } \\
\text { now }\end{array}$ & $\begin{array}{l}\text { COVID-19: Effective } \\
\text { Nursing in Times of } \\
\text { Crisis }\end{array}$ & Online course & $\begin{array}{l}2 \text { weeks - } \\
2 \text { hours } \\
\text { per week }\end{array}$ & $\begin{array}{l}\text { Johns Hopkins School } \\
\text { of Nursing }\end{array}$ \\
\hline $\begin{array}{l}\text { Available } \\
\text { now }\end{array}$ & $\begin{array}{l}\text { WHO Academy and } \\
\text { WHO Info mobile } \\
\text { applications }\end{array}$ & Mobile app & & WHO \\
\hline $\begin{array}{l}\text { Available } \\
\text { now }\end{array}$ & $\begin{array}{l}\text { COVID-19: } \\
\text { Pandemics, } \\
\text { Modelling and Policy }\end{array}$ & Online learning & $\begin{array}{l}2 \text { weeks | } \\
2 \text { hours } \\
\text { weekly } \\
\text { study }\end{array}$ & $\begin{array}{l}\text { FutureLearn UNESCO } \\
\text { UNITWIN Complex } \\
\text { Systems Digital } \\
\text { Campus/Open } \\
\text { University }\end{array}$ \\
\hline
\end{tabular}




\begin{tabular}{|c|c|c|c|c|}
\hline 11.5 .2020 & $\begin{array}{l}\text { COVID-19 Contact } \\
\text { Tracing course }\end{array}$ & Online learning & 5 hours & $\begin{array}{l}\text { Johns Hopkins } \\
\text { Bloomberg School of } \\
\text { Health }\end{array}$ \\
\hline $\begin{array}{l}7-28 \text { May } \\
2020\end{array}$ & $\begin{array}{l}\text { Virtual Evidence } \\
\text { Weeks }\end{array}$ & 5 sessions & $1 \mathrm{~h} 30$ & $\begin{array}{l}\text { International Initiative } \\
\text { for Impact Evaluation } \\
\text { (3ie) }\end{array}$ \\
\hline $\begin{array}{l}\text { Tuesdays } \\
\text { at } 1700 \\
\text { CEST } \\
\text { (Geneva } \\
\text { time) \& } \\
\text { Thursdays } \\
\text { 0830 CEST } \\
\text { (Geneva } \\
\text { time) }\end{array}$ & $\begin{array}{l}\text { COVID-19 Open } \\
\text { online brief with Dr } \\
\text { David Nabarro }\end{array}$ & Event & $1 \mathrm{~h}$ & $4 S D$ \\
\hline $\begin{array}{l}\text { Available } \\
\text { now }\end{array}$ & $\begin{array}{l}\text { Emerging respiratory } \\
\text { viruses, including } \\
\text { COVID-19: methods } \\
\text { for detection, } \\
\text { prevention, response } \\
\text { and control }\end{array}$ & Online learning & 3 hours & WHO \\
\hline $\begin{array}{l}\text { Available } \\
\text { now }\end{array}$ & $\begin{array}{l}\text { Responding to } \\
\text { COVID-19: Real-time } \\
\text { training for the } \\
\text { coronavirus disease } \\
\text { outbreak }\end{array}$ & Online learning & $\begin{array}{l}\text { Multiple } \\
\text { self-paced } \\
\text { course }\end{array}$ & WHO \\
\hline $\begin{array}{l}25 \text { May } \\
2020\end{array}$ & $\begin{array}{l}\text { COVID-19: Tackling } \\
\text { the Novel } \\
\text { Coronavirus }\end{array}$ & Online learning & $\begin{array}{l}3 \text { weeks | } \\
4 \text { hours } \\
\text { weekly } \\
\text { study }\end{array}$ & $\begin{array}{l}\text { FutureLearn } \\
\text { LSHTM/UK PHRST }\end{array}$ \\
\hline $\begin{array}{l}\text { Available } \\
\text { online now } \\
\text { without } \\
\text { mentors. } \\
\text { Updated } \\
\text { version will } \\
\text { commence }\end{array}$ & $\begin{array}{l}\text { COVID-19 } \\
\text { Diagnostics and } \\
\text { Testing }\end{array}$ & Online learning & $\begin{array}{l}3 \text { weeks | } \\
3 \text { hours } \\
\text { weekly } \\
\text { study }\end{array}$ & $\begin{array}{l}\text { FutureLearn } \\
\text { FIND/LSHTM/ASLM }\end{array}$ \\
\hline
\end{tabular}




\begin{tabular}{|l|l|l|l|l|}
$\begin{array}{l}\text { early June } \\
2020\end{array}$ & & & \\
\hline 6 April 2020 & $\begin{array}{l}\text { COVID-19 Critical } \\
\text { Care: Understanding } \\
\text { and Application }\end{array}$ & Online learning & $\begin{array}{l}5 \text { weeks } \\
1 \text { hour } \\
\text { weekly } \\
\text { study }\end{array}$ & $\begin{array}{l}\text { FutureLearn University } \\
\text { of Edinburgh \& Royal } \\
\text { College of Physicians } \\
\text { of Edinburgh }\end{array}$ \\
\hline $\begin{array}{l}\text { Available } \\
\text { now }\end{array}$ & $\begin{array}{l}\text { COVID-19 supporting } \\
\text { online courses }\end{array}$ & Online learning & $\begin{array}{l}\text { Multiple } \\
\text { self-paced } \\
\text { course }\end{array}$ & BMJ Learning \\
\hline
\end{tabular}

\section{Suggested citation}

Millington, K.A. (2021). COVID-19 Health Evidence Summary No.114. K4D Evidence Summary. Brighton, UK: Institute of Development Studies. DOI: 10.19088/K4D.2021.027

\section{Rapid review methodology}

The rapid weekly search for peer-reviewed literature is carried out through a PubMed search with the following keywords ("COVID-19" OR "severe acute respiratory syndrome coronavirus 2" OR "2019-nCoV" OR "SARS-CoV2" OR "2019nCoV" OR "coronavirus" ) AND ("Africa" OR "South Asia" OR "Developing" OR "low-income" OR "low income" OR "lower-middle income" OR "low and middle income" OR "LMIC" OR "LIC" OR "global south") OR ("poverty") OR ("equity" OR "equities"), restricted to articles published in the previous 2 to 3 days, in English. This is complemented by a search of the homepage of the following high-impact global health journals: The Lancet journals, New England Journal of Medicine, Nature, JAMA, Annals of Internal Medicine, Cochrane Reviews, BMJ Global Health, the PLoS journals and a Twitter search of their Twitter pages. A search also of preprints from bioRxiv and medRxiv. Please note that papers that have not been peer-reviewed are highlighted in red. All primary research papers that relate to the primary and secondary impacts of the COVID-19 response in LMICs, and disease control and health system responses are included. Articles related to tackling the secondary impacts on other sectors are not included. Additional commentaries, opinions, and commissioned pieces are selected based on relevance.

The search for dashboards, guidelines, tools, editorials, comments, blogs, opinions and news is through the academic journals listed above, C19 resource hubs and following lead academics and professionals on Twitter. 


\section{About this report}

This weekly COVID-19 health evidence summary (HES) is based on 3.5 hours of desk-based research. The summary is not intended to be a comprehensive summary of available evidence on COVID-19 but aims to make original documents easily accessible to decision makers which, if relevant to them, they should go to before making decisions. The HES are not intended to replace medical or professional advice and the researcher or the K4D consortium cannot be held responsible for any decisions made about COVID-19 on the basis of the HES alone. K4D services are provided by a consortium of leading organisations working in international development, led by the Institute of Development Studies (IDS), with Education Development Trust, Itad, University of Leeds Nuffield Centre for International Health and Development, Liverpool School of Tropical Medicine (LSTM), University of Birmingham International Development Department (IDD) and the University of Manchester Humanitarian and Conflict Response Institute (HCRI).

This evidence summary was prepared for the UK Government's Foreign, Commonwealth and Development Office (FCDO) and its partners in support of pro-poor programmes. Except where otherwise stated, it is licensed for non-commercial purposes under the terms of the Open Government Licence v3.0. K4D cannot be held responsible for errors, omissions or any consequences arising from the use of information contained in this health evidence summary. Any views and opinions expressed do not necessarily reflect those of FCDO, K4D or any other contributing organisation.

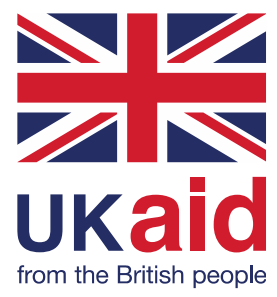

(c) Crown copyright 2021 